\section{Free educational}

\section{material}

Toothfriendly International, a non-profit organisation based in Switzerland, is offering UK dental practices a pack of free educational material and product samples to support their Smile Month initiatives.

Toothfriendly International is nonprofit association with a network of preventive-minded dentists, institutions in dental and public health, the confectionery industry and manufacturers of oral care products.

National Smile Month, (May 13 to June 12 ) is the biggest oral health campaign carried out by thousands of preventionminded dental professionals throughout the country. This year the theme is 'Two Minutes Twice a Day'.

The aim of the Toothfriendly resource pack is to promote the consumption of healthier snacks - without compromising on taste. There is a wide range of Toothfriendly confectionery to choose from, and the "Happy Tooth" logo helps to identify products which are guaranteed safe for teeth.

The Toothfriendly Smile Month pack contains patient leaflets and a yummy selection of sugar-free freebies to be distributed to your patients.

To claim your free resource pack, please send your name, job title, delivery address, telephone number and email details to Kati Leskinen at contact@toothfriendly. ch by the end of April.

Reader response number 51

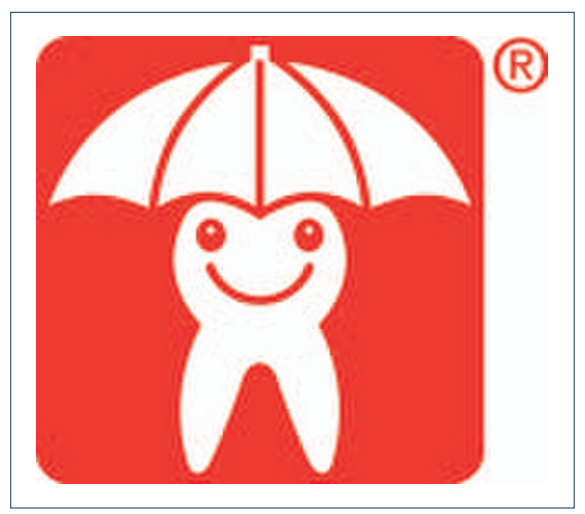

\title{
Silent oil free compressor
}

A new silent and oil free dental air compressor is available with the launch of the silent VTS oil free range, from Bambi. Not just an acoustic hood, the dedicated range of compressors is engineered with design in mind too. Based on the recently launched VT range, this new range has been designed with temperature controlled cooling fans to ensure the compressor runs efficiently with reliability and noise levels from just 54dB(A).

Comprehensive specification is part of every Bambi compressor; internally coated air receivers to ensure against corrosion, a soft start valve which enables the compressor to be turned off remotely without causing harm and an

\section{Birthday celebrations}

The Benevolent Fund has begun birthday celebrations to mark its 125 th year. The Benevolent Fund is a registered charity, providing financial assistance from voluntary contributions to assist dentists and their dependants, when they find themselves in need. It may be an elderly dentist or widow, who finds that the value of their savings and pension has dwindled, or a younger dentist who cannot work because of illness, stress or depression. The only requirement the Fund makes is that at some time the dentist has been on the UK Dental Register.

Throughout the whole of 2007 the Benevolent Fund will be remembering the past 125 years of its charitable

\section{Stylish dental loupe}

Keeler has introduced a new, stylish frame for its range of dental loupes. The new frames wrap around and grip the head so that their weight is more evenly distributed, making them feel lighter.

They also have a stylish, modern look and come in a range of metallic colours including sky blue, navy blue, red and black.

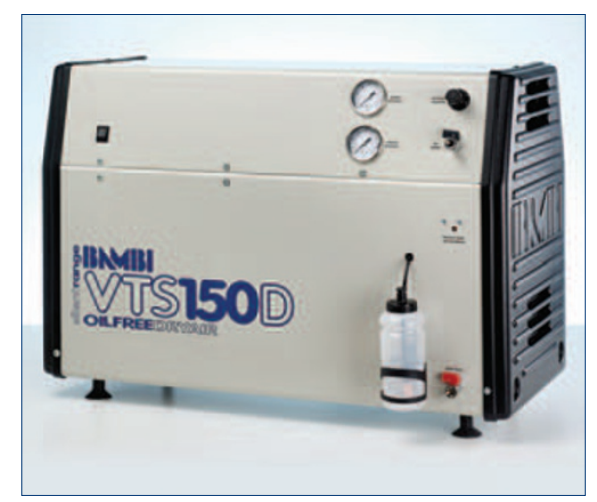

air dryer option are all incorporated across the range.

Reader response number 50

success with the support of many other organisations, including the Association of Specialist Providers to Dentists (ASPD). The ASPD is an association of reputable businesses that offer professional services tailored to meet the specific needs of dentists and the dental industry and during 2007 members of the ASPD will be testing their endurance by undertaking a massive $125 \mathrm{~km}$ sponsored bike ride to celebrate 125 years of the Benevolent Fund.

Established for dentists by dentists, the Benevolent Fund relies solely on support from the dental profession to fund its activities, and its many supporters are only too happy to help in its very special 125 th year.

Reader response number 52

Loupes and lights are easily attached to the front of the frames allowing them to be fixed or removed whenever necessary. An extended, protective side shield protects the user's eyes from injury by pieces of broken teeth during drilling.

The shield also helps to prevent crossinfection from the patient's mouth when water sprays or aerosols are used.

Reader response number 53 

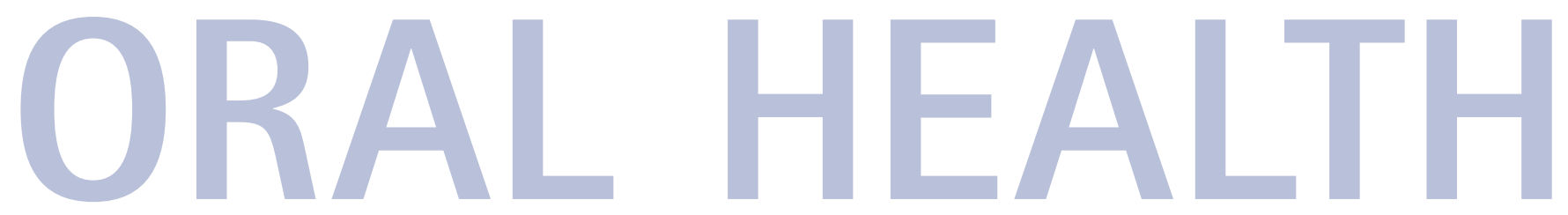

\section{Plaque removal tool}

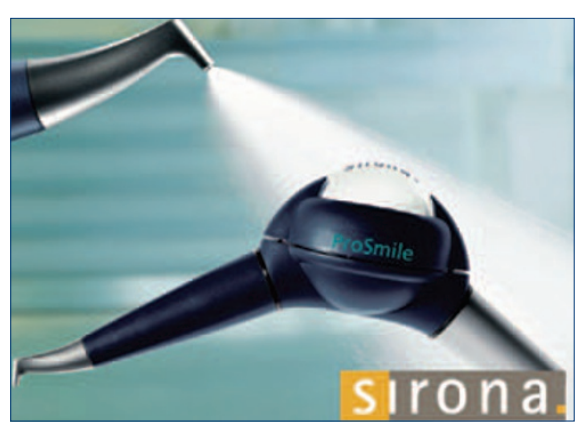

The ProSmile Handy tool from Sirona provides an effective method of prophylactic air abrasive polishing for removal of plaque from all tooth surfaces. Patients can also enjoy a pleasant minty taste during treatment.

The ProSmile Handy is completely adjustable and can be used with an interchangeable $90^{\circ}$ or $120^{\circ}$ angle spray tip for optimum access, and there is a large capacity chamber to provide adequate powder supply for more than one patient.

Its design ensures a balanced and relaxed operation by the user and can be used for the effective and minimally invasive removal of plaque to the patient.

It comes with the Sirona Click \&t Go interface allowing the unit to be attached to most popular brands of handpiece couplings.

Reader response number 55

\section{Skin-friendly moisturising gloves}

Ansell Healthcare have recently introduced a new latex glove specifically intended for double gloving and which incorporates HydraSoft Technology. Concerned by the high rate of infections amongst medical personnel, Ansell has launched a campaign to promote the use of double gloving for surgical procedures.

As thin and comfortable as a classical Gammex PF HydraSoft glove, the dark green Gammex PF Underglove features a moisturising HydraSoft coating to help promote healthy, unbroken skin. Integrated in the gloves' inner lining, HydraSoft Technology contains a neu- tral, odourless skin-friendly moisturiser combined with Dimethicone, which forms a protective barrier that blocks attacks from foreign substances and prevents the skin from drying out.

A polyurethane lining with hydrophilic and hydrophobic elements allows easy damp donning and quick intra-operative glove changing. Gammex PF Underglove is manufactured using Protein and Endogenous Allergen Reduction Leaching (P.E.R.L.) Technology that delivers a glove with extremely low extractible proteins, reducing risk of latex sensitisation. Reader response number 54

\section{Effective cleaning in interdental spaces}

Curaprox manufactures a large number of interdental brushes designed specifically

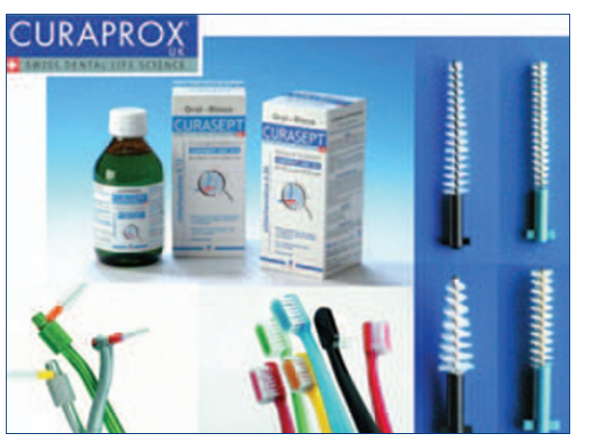

to effectively penetrate the interdental spaces that are most at risk. Curaprox offer an autoclavable IAP (Interdental Access Probe) and card which allows clinicians to identify to the patient the correct brush size for each interdental space.

A new, SR (Straight Ready) brush range, complementing the existing range, is now available which is instantly ready for use. This new range comes in five colour coded widths to adapt to the needs of every patient, including turquoise $0.4-4.5 \mathrm{~mm}$ and pink $0.6 \mathrm{~mm}$.

Reader response number 56

\section{Blended mix gloves}

Bodyguards Vitrile powder free synthetic gloves are the newest addition to the Bodyguards comprehensive glove range from Brosch direct. The new Vitrile gloves are manufactured from a blended mix of Vinyl and Nitrile compounds to offer the best advantages of both materials.

Softer, more flexible, with greater sensitivity and less cost than Nitrile, the new Vitrile gloves are designed to offer a solution to users of synthetic gloves who prefer a stronger material and better fit compared to Vinyl.

The Bodyguards glove range is one of the largest imported glove brands and one of the most comprehensive ranges available in the UK. All medical gloves are manufactured to BS EN 455 medical standard.

Reader response number 57

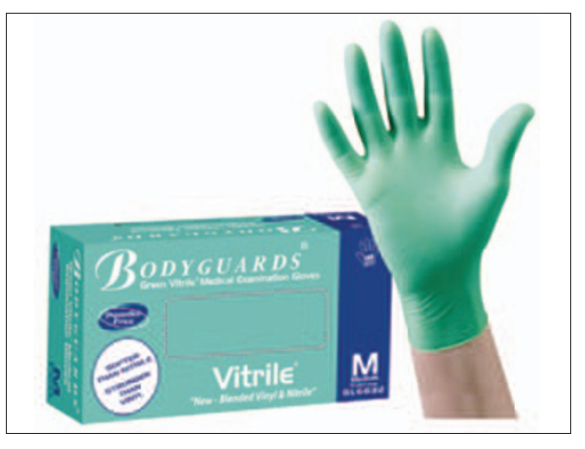




\section{Accelerating natural healing}

Gengigel from Oraldent has recently been accepted in the British National Formulary (BNF). As it is on the Drug Tarrif Part IX, Gengigel is now listed on the BNF under 'oral ulceration and inflammation'.

Previously only doctors were authorised to prescribe Gengigel Rinse $150 \mathrm{ml}$ and Gengigel Gel $20 \mathrm{ml}$, but with this recent acceptance, it has been confirmed that dentists now have the authorisation to also prescribe both the gel and the rinse, indicating which form of Gengigel the patients require.

Gengigel products accelerate the natural healing processes of mouth tissue and contain the active ingredient hyaluronan, which when introduced to the mouth can accelerate tissue healing, repair and regeneration. Hyaluronan controls the interaction between extracellular and intercellular

\section{Patient protection bib}

Henry Schein are sole suppliers of CollarDam, the new patient protection bib. Designed for dentists by a dentist, the bibs give each patient a high-quality, spa like experience.

With traditional bibs patients can feel wet, cold and stressed. They are uncomfortable, especially during long

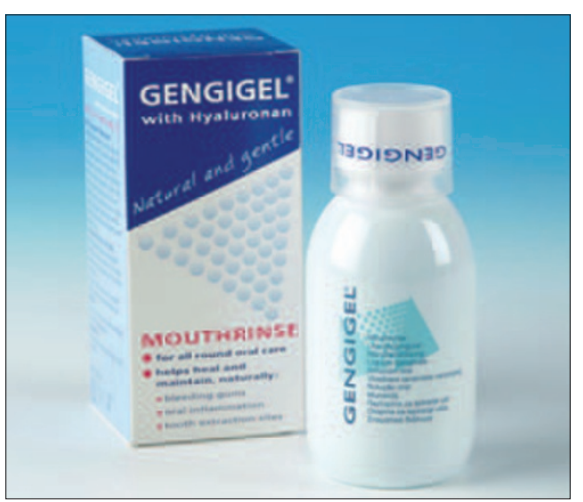

structures and helps to reduce inflammatory cytokine interactions; it is a natural substance, which performs a number of structural and physiological functions within the tissues. It also acts to regulate osmotic pressure and tissue lubrication.

Reader response number 58

\section{Larger than life}

Trycare Dental Linkline supplies a wide range of oral health products. The SDI Directa Demonstrator Model and Toothbrush are larger than life replicas that enable you to give chairside instruction and demonstration of tooth brushing techniques. Their size makes it easy to demonstrate, clearly, the subtleties of good oral hygiene procedure.

CCS Prophy Paste is colour coded for easy identification of grit size and indication. The coarse blue paste is used on heavily discoloured surfaces and thick plaque layers; the medium green paste on moderately thick plaque and discoloured teeth; the fine red universal paste on smaller amounts of plaque, slightly discoloured teeth and root surfaces; the extra fine yellow paste for polishing teeth and restorations, leaving an extremely smooth finish.

Non-splattering, CCS Prophy Paste does not dry out, liquefy or separate and has a long shelf life. Easy to remove, it replaces fluoride when polished off and does not leave an oily residue. procedures. With ground-breaking super-absorbent fibre technology, the high-tech, patented design is a combination of crystals and fibre that changes colour when wet to indicate the bib should be changed, a further guarantee of the patients' comfort. The bib itself also acts as one large tissue and is ideal for dabbing.

Reader response number 59

Rondell Disclosing Pellets provide an easy way to detect plaque. Simply rub the tooth surface with the pre-soaked pellets and plaque appears. Rondell Red highlights 3 day old plaque. Rondell Blue highlights plaque that is older. Both are easily brushed off, leaving a mild peppermint taste.

Reader response number 60

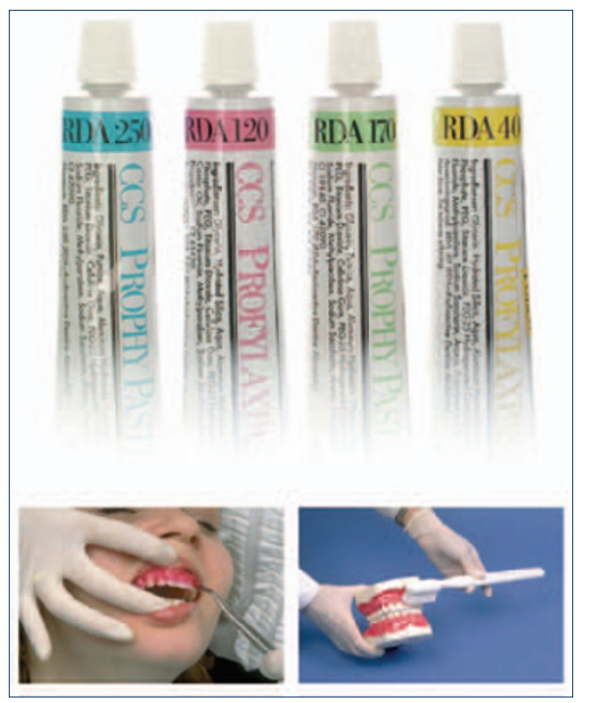

